\title{
Umbelliferone as a Small Molecular Peroxidase Mimic towards Sensitive Detection of $\mathrm{H}_{2} \mathrm{O}_{2}$ and Glucose
}

\author{
Chaoqun Sun, Zili Huang, Li LiU, Menglu Li, and Huzhi ZHENG ${ }^{\dagger}$ \\ Key Laboratory of Luminescent and Real-Time Analytical Chemistry (Southwest University), Ministry of Education, \\ College of Chemistry and Chemical Engineering, Southwest University, Beibei, Chongqing 400715, China
}

\begin{abstract}
In this work, umbelliferone, a kind of coumarin derivative, was proved to exhibit peroxidase-like activity that could catalyze the oxidation of $3,3^{\prime}, 5,5^{\prime}$-tetramethylbenzidine (TMB) in the presence of hydrogen peroxide to generate a blue-colored oxide (oxTMB). The catalytic mechanism is similar to that of native enzymes (e.g. horseradish peroxidase, HRP) and nanozymes, which follow the Michaelis-Menten kinetics behavior. Meanwhile, the 7-hydroxyl group of umbelliferone plays a significant role in the peroxidase-like activity. Compared with enzymes and nanozymes, this small molecular mimic enzyme possesses the advantages of low cost, simple molecular structures, small molecular weight and high stability against harsh conditions. Based on the favorable peroxidase mimetic activity of umbelliferone, a convenient, practical and sensitive $\mathrm{H}_{2} \mathrm{O}_{2}$ and glucose detection method was successfully established. This work not only opens some new inspirations into seeking for novel molecular enzyme mimetics with excellent catalytic activities, but also provides promising assays for clinical diagnosis.
\end{abstract}

Keywords Umbelliferone, 3,3',5,5'-tetramethylbenzidine, peroxidase mimic, glucose, chromogenic reaction

(Received January 17, 2018; Accepted April 11, 2018; Published August 10, 2018)

\section{Introduction}

Natural enzymes are biological catalysts; although a few are RNA molecules, most enzymes are generally proteins that increase the rate of a chemical reaction by lowering their activation energy and make their conversion of substrate to product occur many millions of times faster. ${ }^{1}$ Due to their special selectivity, high catalytic efficiency occurs under ecofriendly reaction conditions; ${ }^{2}$ natural enzymes have recently been applied to the chemical industry, biotechnology, environmental analysis, ${ }^{3}$ especially to biosensing and immunoassays. ${ }^{4}$ Although natural enzymes are highly active and specific during their catalytic behavior, their expensive cost, difficult purification process, low operation stability and rigorous storage conditions hamper their extensive applications. Hence, increasing attention has been paid by researchers to discovering and constructing enzyme mimics with similar functions to that of natural enzymes. ${ }^{5}$ Since Yan's group reported on the intrinsic peroxidase-like activity of ferromagnetic nanoparticles in $2007,{ }^{6}$ a series of nanomaterial-based artificial enzymes, including metal nanoparticles, ${ }^{7-9}$ metal oxides and their combined nanostructures, ${ }^{10-12}$ and carbon-based materials, ${ }^{13}$ have also been affirmed to have peroxidase-like activities during the past few decades. They occupied excellent superiorities over traditional natural enzymes, such as easy preparation and storage, design flexibility and good stability under diversified conditions. Unfortunately, dissatisfied activity differences from batch to batch, easy aggregation with high concentrations have

† To whom correspondence should be addressed.

E-mail: zhenghz@swu.edu.cn seriously restricted their comprehensive utilization. ${ }^{14}$

Therefore, that is of vital importance to find substitutes with remarkable catalytic activity similar to that of natural enzymes and nanozymes. Our group had found that fluorescein exhibits noteworthy peroxidase-like activity. ${ }^{15}$ This opens small molecules as peroxidase mimics, and provides some new inspirations into seeking for novel molecular enzyme mimics with excellent catalytic activities. In addition, we also investigated the peroxidase-like activity of other xanthene dyes with similar structure to fluorescein, such as rhodamine B, rhodamine $6 \mathrm{G}$, and found that their xanthene rings without any hydroxyl groups did not possess peroxidase-like activity. ${ }^{15}$ Based on this result, we may infer the hydroxyl groups could play a crucial role in the catalytic systems. Therefore, we speculated that other substances with hydroxyl groups and similar molecular structure to fluorescein perhaps have the same activity. Herein, we found another kind of small molecules, umbelliferone, a kind of coumarin derivative that possess intrinsic peroxidase-like activity in the catalytic oxidation of peroxidase substrate $3,3^{\prime}, 5,5^{\prime}$-tetramethylbenzidine (TMB) in the presence of $\mathrm{H}_{2} \mathrm{O}_{2}$ (illustrated in Scheme 1).

Umbelliferone is also known as 7-hydroxycoumarin, displayed in Scheme 1, which is derived from the plant family Umbelliferae ${ }^{16}$ and is present in many plants, such as carrot, coriander and garden angelica. ${ }^{17}$ This compound is also commercially available in large quantities. ${ }^{18}$ Meanwhile, it possesses a wide range of bioactivities such as anti-inflammatory, antitumor promoting and antiproliferative. ${ }^{19,20}$ Further, this compound is also the main metabolite of coumarin with strong fluorescence, and has served as a core to prepare a range of coumarin derivatives for extensive applications. ${ }^{21}$ However, to the best of our knowledge, its catalytic activity has not been 


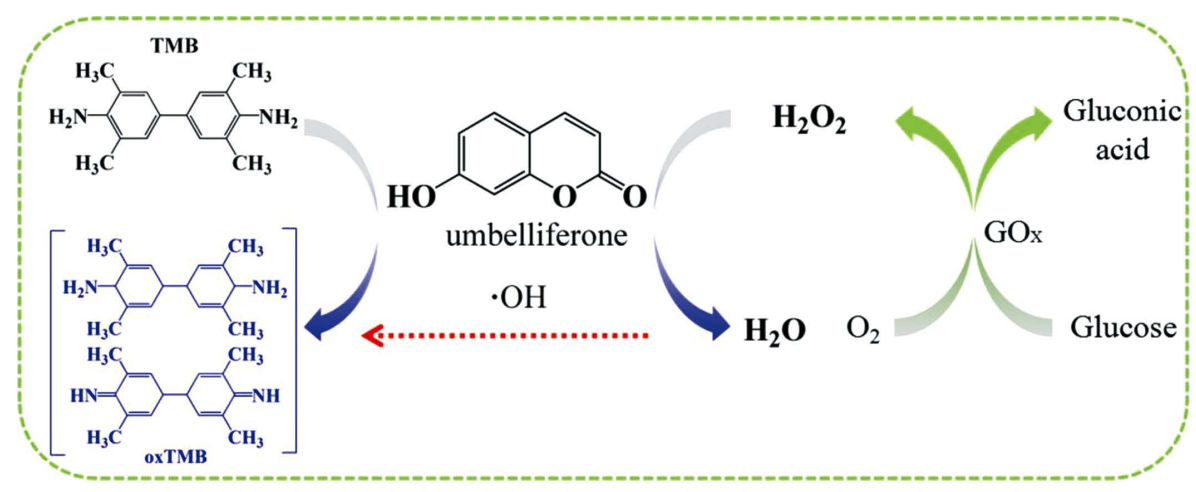

Scheme 1 Schematic illustration of a peroxidase-like catalytic reaction of umbelliferone for colorimetric detection of $\mathrm{H}_{2} \mathrm{O}_{2}$ and glucose by coupling with glucose oxidase (GOx).

reported so far. Herein, we demonstrated umbelliferone to have good peroxidase-like catalytic performance. Compared with natural enzymes and some artificial nanozymes, ${ }^{22}$ umbelliferone is a particularly efficient catalyst owing to its high abundance, low cost, remarkable thermal stability and good tolerance of high $\mathrm{H}_{2} \mathrm{O}_{2}$ concentrations and strong acid or alkali environments. At the same time, umbelliferone, with a simple and similar molecular structure, low molecular weight and fast reaction time, is better than fluorescein. Hence, umbelliferone could have great potential as an ideal candidate for biomimetic catalysis.

In the present work, the mechanism, optimal reaction conditions, catalytic kinetics, stability and robustness under harsh conditions of the peroxidase-like activity of umbelliferone were considered. Furthermore, we investigated the effect of the 7-hydroxyl group on its molecular structure to the peroxidaselike activity. ${ }^{23}$ Subsequently, in view of the excellent peroxidaselike activity of umbelliferone, we successfully established a convenient, brief and sensitive $\mathrm{H}_{2} \mathrm{O}_{2}$ and glucose detection system. This system will provide some novel ideas concerning practical chemical analysis.

\section{Experimental}

\section{Reagents and chemicals}

Umbelliferone (Umb, GC grade, $\geq 98 \%$ ), coumarin (Cou, GC grade, $99.0 \%$ ) and esculetin (Esc, HPLC grade, 98.0\%) were purchased from TCI Chemical Industry (Tokyo, Japan). 4,7-Dihydroxycoumarin (4,7-DHC, 97.0\%), glucose oxidase (GOx, EC1.1.3.4.47, $200 \mathrm{U} / \mathrm{mg}$ ) and 3,3',5,5'-tetramethylbenzidine (TMB) (99\%) were purchased from Sigma-Aldrich. 5,7-Dihydroxycoumarin (5,7-DHC, 98.0\%) was purchased from Ark Pharm, Inc. (Chicago, USA). $\mathrm{H}_{2} \mathrm{O}_{2}(30 \%)$ and glucose were purchased from Chongqing Chuandong Chemical Co. Ltd. (Chongqing, China). Superoxide dismutase (SOD, $2500 \mathrm{U} / \mathrm{mg}$ ) was purchased from Sangon Biotech (Shanghai) Co., Ltd. Acetate (HPLC grade, $\geq 99.9 \%$ ) was obtained from Shanghai Mackin biochemical Co. Ltd. (Shanghai, China). Sodium acetate anhydrous $(99.99 \%$, metals basis) and sodium azide $(99.5 \%)$ were obtained from Aladdin (Shanghai, China). All other reagents were of analytical grade, and used without further purification. All aqueous solutions were prepared using Milli-Q water (Millipore, USA) throughout the study.

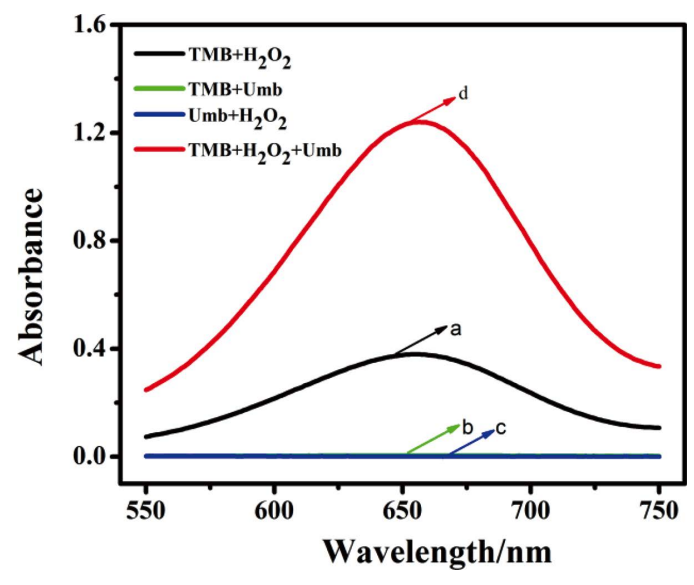

Fig. 1 Absorption spectra of different catalytic reaction systems: (a) $\mathrm{TMB}+\mathrm{H}_{2} \mathrm{O}_{2}$ (black line), (b) $\mathrm{TMB}+\mathrm{Umb}$ (green line), (c) $\mathrm{Umb}+\mathrm{H}_{2} \mathrm{O}_{2}$ (blue line), (d) $\mathrm{TMB}+\mathrm{H}_{2} \mathrm{O}_{2}+\mathrm{Umb}$ (red line).

\section{Results and Discussion}

\section{Peroxidase mimetic activity of umbelliferone}

To examine the peroxidase-like activity of umbelliferone, the catalytic oxidation of the peroxidase substrate TMB in the absence or presence of $\mathrm{H}_{2} \mathrm{O}_{2}$ was implemented, and the absorption spectra were monitored. The characteristic bluecolored products (oxTMB) had typical absorption peaks at $652 \mathrm{~nm}$ stemming from the distinctive blue charge-transfer complex between di-amine and di-imine. ${ }^{24}$ It can be easily observed from Fig. 1 and Fig. S1, that the TMB $+\mathrm{H}_{2} \mathrm{O}_{2}$ system revealed a light-blue color with a low absorption peak at $652 \mathrm{~nm}$ (curve a, Fig. 1); the TMB $+\mathrm{Umb}$ or $\mathrm{Umb}+\mathrm{H}_{2} \mathrm{O}_{2}$ system was almost colorless and the absorbance at $652 \mathrm{~nm}$ was near zero (curves $\mathrm{b}$ and c, Fig. 1), while the TMB $+\mathrm{H}_{2} \mathrm{O}_{2}+\mathrm{Umb}$ system showed a dark-blue color with an obvious absorption peak (curve $d$, Fig. 1). The chromogenic reaction catalyzed by Umb showed an observably higher reaction velocity than the reaction catalyzed only by $\mathrm{H}_{2} \mathrm{O}_{2}$ (Fig. S2). This indicated the catalytic oxidation of TMB derived from the contribution of Umb. Moreover, it was found that the catalytic reaction system is dependent on the concentrations of umbelliferone. As shown in Fig. S3, with the increase of the concentrations of umbelliferone, the absorbance value at $652 \mathrm{~nm}$ was gradually raised. The results both further testified that Umb acts as a peroxidase 
mimic toward TMB. To further characterize the peroxidase-like activity of Umb, we repeated the experiments using other peroxidase substrates in place of TMB, including $o$-phenylenediamine (OPD) and 2,2'-azino-bis(3-ethylbenzothiazoline-6sulfonic acid) (ABTS). As shown in Fig. S4, that Umb not only catalyzes the oxidation of TMB producing a blue color, but also OPD to given an orange color and ABTS to given a green color. These results indicate that Umb has same peroxidase-like activity if other colorimetric substrates, such as OPD and ABTS, are used.

\section{Optimization of reaction conditions}

Similar to HRP and nanozymes, ${ }^{25}$ the peroxidase-like activity of Umb was dependent on the $\mathrm{pH}$, temperature, $\mathrm{H}_{2} \mathrm{O}_{2}$ concentrations and reaction time. To confirm this, the reaction conditions of Umb and HRP were conducted varying $\mathrm{pH}$ value from 1.0 to 7.0 , the temperature from 15 to $60^{\circ} \mathrm{C}$, the $\mathrm{H}_{2} \mathrm{O}_{2}$ concentrations from 0 to $6.0 \mathrm{mM}$ and the reaction time from 15 to $180 \mathrm{~min}$. As displayed in Fig. S5a, the absorbance increased with the $\mathrm{pH}$ and reached a maximum at $\mathrm{pH} 3.0$, but then dropped down. It is worth noting that the molecular structure of umbelliferone constantly changed under different pH conditions, similar to hymecromone. ${ }^{26}$ As shown in Fig. S6, in the strong acid environment, the carbonyl group of umbelliferone was protonated ${ }^{24}$ (inset in type I), which increased the degree of conjugation of benzopyrone and contributed to the peroxidaselike activity of umbelliferone. Meanwhile, over a range of pH $3-6$, the carbonyl group of umbelliferone was gradually deprotonated, which exists as a neutral molecule ${ }^{27}$ (inset in type II), the peroxidase activity was gradually reduced; when the $\mathrm{pH}$ was continually raised, the hydroxyl group of umbelliferone was ionized to become an anion and the pyran ring was open, which deceased the degree of conjugation and rigidity of umbelliferone ${ }^{27}$ (inset in type III, IV). This could explain that during the ionization and hydrolyzation process, the peroxidaselike activity of umbelliferone is gradually changed and the maximum catalytic activity is obtained at $\mathrm{pH}$ 3.0.

Similarly, as shown in Fig. S5, the catalytic activity of umbelliferone with the change of the other reaction conditions constantly fluctuate and the maximum activity was obtained under the optimal reaction conditions: temperature $50^{\circ} \mathrm{C}$ (Fig. S5b), $\mathrm{H}_{2} \mathrm{O}_{2}$ concentrations $4.0 \mathrm{mM}$ (Fig. S5c) and reaction time 90 min (Fig. S5d). The optimal condition was similar to that of natural enzyme (e.g. HRP). What is more, compared to HRP, umbelliferone exhibited remarkable tolerance when it was situated in high $\mathrm{H}_{2} \mathrm{O}_{2}$ concentrations, which could establish extensive applications under various conditions.

\section{The robustness of umbelliferone under harsh environment}

The influences on the peroxidase mimetic catalytic activity of umbelliferone while being placed in different temperatures and $\mathrm{pH}$ environments for some time were explored. It was expected to be more stable than natural enzymes (e.g. HRP). First, we incubated Umb and HRP within a range of $\mathrm{pH}$ values $(\mathrm{pH} 1-12)$ or different temperatures in a range of $4-90^{\circ} \mathrm{C}$ for $2 \mathrm{~h}$, and then the activities were measured under the optimal conditions, as described in supplementary information (SI). It can be observed from Fig. S7a that the relative activity was almost retained at more than $65 \%$ over the range of $\mathrm{pH}$ from 1 to 12 . Especially, with a change of the $\mathrm{pH}$, the catalytic activity of umbelliferone was gradually reduced until equilibrium occurred. Meanwhile, as displayed in Fig. S7b, the umbelliferone kept almost $90 \%$ of the peroxidase-like activity over a wide range of temperatures from 4 to $90^{\circ} \mathrm{C}$. However, the results showed that the activity of HRP was largely inhibited after incubation at lower or higher
$\mathrm{pH}$ or temperatures over $40^{\circ} \mathrm{C}$. These directly demonstrated the robust stability of umbelliferone as a small molecular enzyme mimic, compared to natural enzyme.

\section{Reactive species analysis}

To confirm the generation of reactive species during the catalytic process, reactive species trapping experiments were carried out. ${ }^{28}$ As displayed in Fig. S8, $\cdot \mathrm{OH}$ radical scavengers, such as ascorbic acid (AA), thiourea (TU), caused a dramatic decrease in the absorbance at $652 \mathrm{~nm}$. However, a small change in the absorption occurred after adding ${ }^{1} \mathrm{O}_{2}$ radical scavengers, such as sodium azide $\left(\mathrm{NaN}_{3}\right)$ and $\mathrm{O}_{2^{-}}{ }^{-}$radical scavengers, superoxide dismutase (SOD). The results pointed out that the generation of $\cdot \mathrm{OH}$ radicals played a critical role in oxidizing TMB to produce a blue color.

In addition, in order to further investigate the mechanism of the peroxidase-like activity of umbelliferone, Electron Spin Resonance (ESR) was used to demonstrate the production of free radicals $(\cdot \mathrm{OH})$ during the catalytic reaction procedure using DMPO as a spin trap. ${ }^{29}$ As observed from Fig. S9, weak characteristic peaks can be observed in the DMPO $+\mathrm{H}_{2} \mathrm{O}_{2}$ system. In contrast, a stronger characteristic peak with an intensity ratio of $1: 2: 2: 1$ can be found in the DMPO $+\mathrm{H}_{2} \mathrm{O}_{2}+\mathrm{Umb}$ system, indicating the production of the $\cdot \mathrm{OH}$ radical which is similar to the behavior of peroxidase. ${ }^{30}$ Moreover, with an increase in the concentrations of umbelliferone, the ESR signal intensity increased. This result provides direct evidence that umbelliferone possesses peroxidase mimetic activity.

\section{Steady-state kinetic study}

We performed a kinetic analysis to measure the steady-state kinetic parameters of the chromogenic reaction catalyzed by umbelliferone. Typical Michealis-Menten curves were obtained by drawing the initial reaction velocities against substrate concentrations. The corresponding concentrations of the oxidized peroxidase substrate was obtained by the LambertBeer law using the value $\varepsilon_{\text {oxTMB }}=39000 \mathrm{M}^{-1} \mathrm{~cm}^{-1}$ at $652 \mathrm{~nm}$ based on the absorbance data and the Michaelis-Menten constant was calculated from the Lineweaver-Burk doublereciprocal plot, ${ }^{31,32}$ based on

$$
1 / \nu=K_{\mathrm{m}} / V_{\max } \cdot\left(1 /[\mathrm{S}]+1 / K_{\max }\right) .
$$

As displayed in Fig. 2 (a, b), the reaction rate mounted gradually with increasing TMB or $\mathrm{H}_{2} \mathrm{O}_{2}$ concentrations until achieving the reaction equilibrium. The steady-state kinetic parameters are summarized in Table 1 . The results indicated the catalytic reaction behavior of umbelliferone is similar to that of HRP which followed the Michaelis-Menten kinetics behaviors. The $K_{\mathrm{m}}$ value is represented by the degree of affinity between the enzymes and the substrate: a lower $K_{\mathrm{m}}$ indicates a higher affinity. As can be seen in Table 1, compared with other artificial enzymes, the umbelliferone has a smaller $K_{\mathrm{m}}$ value for $\mathrm{H}_{2} \mathrm{O}_{2}$, which was almost 3-times lower than HRP, ${ }^{15}$ indicating a relatively higher affinity to $\mathrm{H}_{2} \mathrm{O}_{2}$. The $K_{\mathrm{m}}$ value of Umb with TMB was higher than that of HRP. ${ }^{15}$ Figure 2 (c, d) showed double reciprocal plots at different $\mathrm{H}_{2} \mathrm{O}_{2}$ and TMB concentrations, respectively. The lines in each plot are almost parallel, which is the characteristic of a typical ping-pong mechanism, ${ }^{33}$ as was observed for HRP. ${ }^{34}$ This means that the umbelliferone binds and reacts with one substrate (either TMB or $\mathrm{H}_{2} \mathrm{O}_{2}$ ), and then releases a product before reacting with another substrate. 
a)

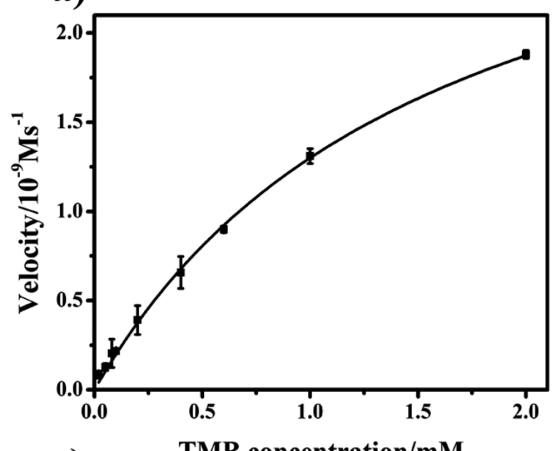

c)

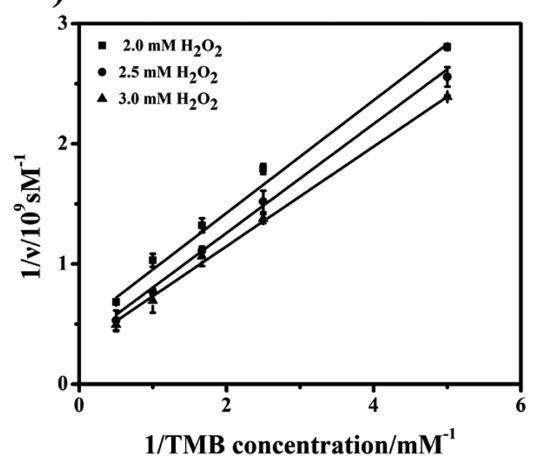

b)
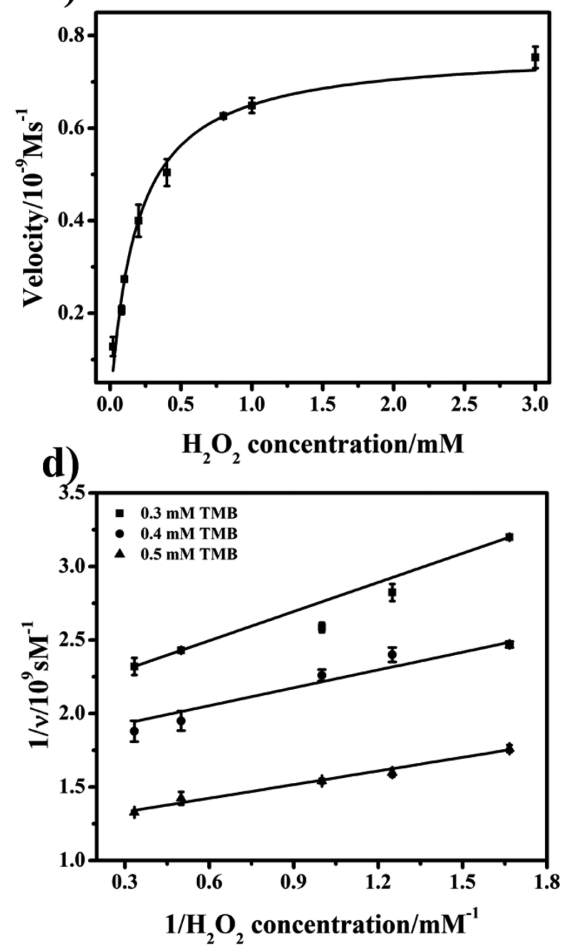

Fig. 2 Steady-state kinetic assay and catalytic mechanism of Umb $(a-d)$.

Table 1 Comparison of the apparent Michaelis-Menten constant $\left(K_{\mathrm{m}}\right)$ and maximum reaction rate $\left(V_{\max }\right)$ between umbelliferone and HRP

\begin{tabular}{lcccc}
\hline Catalyst & Substrate & $K_{\mathrm{m}} / \mathrm{mM}$ & $V_{\text {max }} / 10^{-8} \mathrm{M} \mathrm{s}^{-1}$ & $K_{\text {cat }} / \mathrm{s}^{-1}$ \\
\hline \multirow{2}{*}{$\mathrm{Umb}$} & $\mathrm{TMB}$ & $1.58 \pm 0.132$ & $0.335 \pm 0.016$ & $0.112 \times 10^{-5}$ \\
& $\mathrm{H}_{2} \mathrm{O}_{2}$ & $0.184 \pm 0.01$ & $0.077 \pm 0.001$ & $0.026 \times 10^{-5}$ \\
$\mathrm{HRP}^{15}$ & $\mathrm{TMB}$ & $0.16 \pm 0.025$ & $4.27 \pm 0.456$ & $2.08 \times 10^{2}$ \\
& $\mathrm{H}_{2} \mathrm{O}_{2}$ & $0.52 \pm 0.011$ & $1.82 \pm 0.015$ & $8.02 \times 10^{3}$ \\
\hline
\end{tabular}

The peroxidase mimetic catalytic activity of different umbelliferone analogs

In this work, we demonstrated that $\mathrm{Umb}$ facilitated $\cdot \mathrm{OH}$ radical production by catalyzing the decomposition of $\mathrm{H}_{2} \mathrm{O}_{2}$ to oxide TMB, which is similar to the behavior of fluorescein with intrinsic peroxidase-like activity. ${ }^{15}$ This result may derive from the existence of hydroxyl groups on the Umb. ${ }^{15}$ We suspect that the 7-hydroxyl group of Umb could contributed to catalyze the oxidation of TMB and the peroxidase-like activity perhaps enhance with an increase of the number of hydroxyl groups of Umb. Thus, the peroxidase activities of four umbelliferone analogs were investigated. The differences among umbelliferone analogs are mainly the numbers and location of the hydroxyl groups on the benzopyrone (Fig. S10). As exhibited in Fig. S11a, the results indicated that the Cou (the 7-hydroxyl group of umbelliferone was replaced by hydrogen), has a lower catalytic activity than that of umbelliferone. This conformed preliminarily to our surmise that the existence of the hydroxyl group could play a crucial role in the peroxidase-like activity. Nevertheless, contrary to our guess, along with the increase in the number of hydroxyl groups and the change of the location of the hydroxyl groups on the benzopyrone, the umbelliferone analogs (Esc, 4, 7-DHC and 5, 7-DHC) showed a weak peroxidase-like activity, even less than that of the blank system (without any catalysts). The reasons maybe that the umbelliferone analogs besides coumarin with the numbers of hydroxyl groups increased and the location of the hydroxyl groups changed, acting as antioxidants, and possessed freeradical scavenging properties ${ }^{35}$ that captured the $\cdot \mathrm{OH}$ radical of the reaction system and induced a disappearance of the characteristic absorption peak of oxTMB at $652 \mathrm{~nm}$.

To further investigated this idea, first we carried out the chromogenic reaction of TMB in the assistance of Umb under the optimal conditions; we added different umbelliferone analogs (Esc, 4, 7-DHC and 5, 7-DHC) with different concentrations and incubated for $30 \mathrm{~min}$; finally, we recorded the change of the spectra over 500-800 nm. As shown in Fig. S11 (b, c and d), with the increase of the concentrations of Esc, 4, 7-DHC and 5, 7-DHC, the absorbance at $652 \mathrm{~nm}$ was dramatically decreased. The results manifested that they could act as antioxidants to lead in the fading of oxTMB. Therefore, it demonstrated that with an increase of the numbers of hydroxyl groups, the peroxidase-like activity of umbelliferone analogs is not as good as expected.

\section{Colorimetric detection of $\mathrm{H}_{2} \mathrm{O}_{2}$ and glucose}

As demonstrated above, concerning the peroxidase mimetic properties of umbelliferone being dependent on the $\mathrm{H}_{2} \mathrm{O}_{2}$ concentrations, a colorimetric detection method of $\mathrm{H}_{2} \mathrm{O}_{2}$ was successfully established. Figure $\mathrm{S} 12(\mathrm{a}, \mathrm{b})$ shows the absorption spectra of chromogenic systems in the presence of $\mathrm{H}_{2} \mathrm{O}_{2}$ with different concentrations. The absorbance at $652 \mathrm{~nm}$ was proportional to the $\mathrm{H}_{2} \mathrm{O}_{2}$ concentrations from 1.0 to $100.0 \mu \mathrm{M}$, accompanying the progression of the blue color (inset in Fig. S12b); and the linear regression equation was $A_{652 \mathrm{~nm}}=$ $0.00232 C+0.01197\left(R^{2}=0.9966\right)$ with a detection limit of 
a)

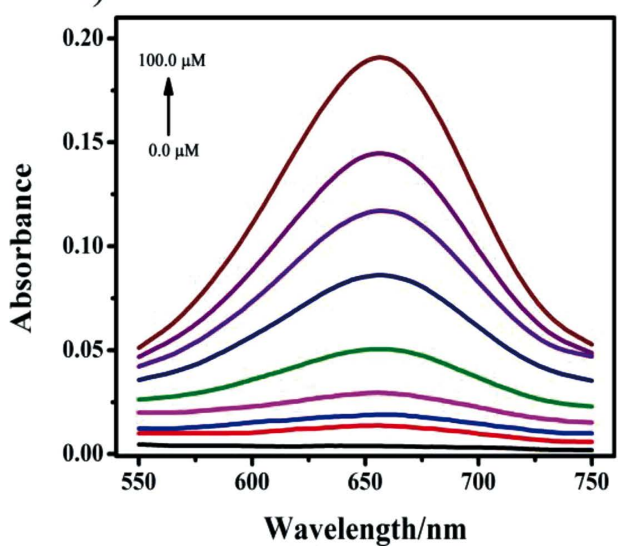

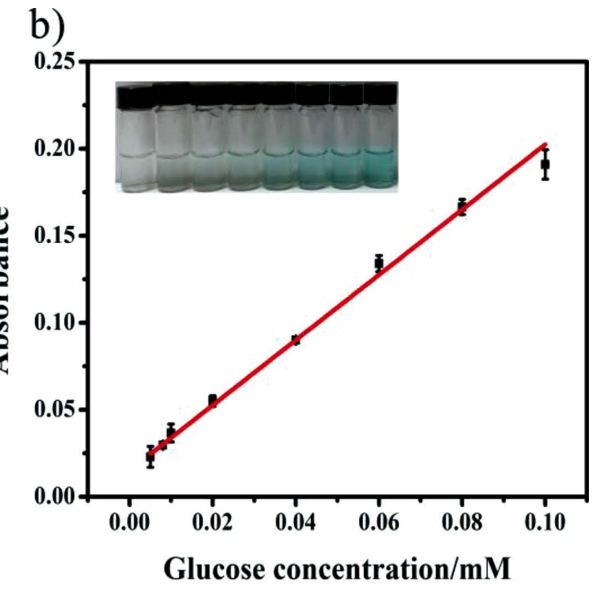

Fig. 3 (a) Typical absorption spectra obtained for the detection of glucose using umbelliferone as a peroxidase mimic under the optimized conditions. (b) Linear calibration plot of glucose detection using umbelliferone as an artificial enzyme.

Table 2 Comparison between the values obtained by our proposed method and hospital for the determination of glucose in serum samples

\begin{tabular}{cccc}
\hline Sample & $\begin{array}{c}\text { Proposed } \\
\text { method/mM }\end{array}$ & $\begin{array}{c}\text { RSD, \% } \\
(n=3)\end{array}$ & $\begin{array}{c}\text { Hospital } \\
\text { result/mM }\end{array}$ \\
\hline 1 & $4.52 \pm 0.05$ & 1.4 & 4.64 \\
2 & $4.65 \pm 0.08$ & 2.4 & 4.71 \\
\hline
\end{tabular}

$0.5 \mu \mathrm{M}(\mathrm{LOD}=3 \sigma / \kappa$ method, in which $\sigma$ means the standard deviation of blank experiment $(n=11)$, and $\kappa$ means the slope of calibration curve), which is comparable to other peroxidase mimic-based methods reported (as shown in Table S1). The good sensitivity to $\mathrm{H}_{2} \mathrm{O}_{2}$-based umbelliferone as a peroxidase mimic was a significant prerequisite for $\mathrm{H}_{2} \mathrm{O}_{2}$-mediated glucose detection by coupling with glucose oxidase (GOx). The glucose could be catalyzed to generate gluconic acid and $\mathrm{H}_{2} \mathrm{O}_{2}$ by GOx in the presence of molecular oxygen, ${ }^{36}$ which is a crucial indicator concerning the diagnosis of diabetes mellitus in clinical medicine. Furthermore, when combined with GOx, a quantitatively colorimetric detection method for glucose can also be established. As shown in Fig. 3, the absorbance at $652 \mathrm{~nm}$ was proportional to the glucose concentrations from 5.0 to $100.0 \mu \mathrm{M}$, and the linear regression equation is $A_{652 \mathrm{~nm}}=$ $1.8727 C+0.0152\left(R^{2}=0.9975\right)$, with a detection limit as low as $2.5 \mu \mathrm{M}$ and the color variation can be observed by naked eyes. The detection limit is comparable to the previous reports of state-of-the-art glucose sensors (as shown in Table S2). To evaluate the selectivity of this proposed system, we utilized fructose, lactose, sucrose and maltose as negative controls. The results (Fig. S13) demonstrated that these glucose analogues were negligible compared with that of glucose, even at concentrations of 5-fold higher (except for the maltose with 2 -fold higher) than that of $0.1 \mathrm{mM}$ glucose. This indicates that this proposed method had good selectivity for glucose, which could be attributed to the high affinity of GOx for glucose. ${ }^{37}$

In order to further demonstrate the practical application of the constructed colorimetric approach for glucose in real samples, two serum samples taken from two healthy subjects in our laboratory workers were used for glucose detection; and the volunteers' consent and approval from the Institutional Research
Ethics Committee of Southwest University hospital were obtained for research purposes. As shown in Table 2, the results obtained by our colorimetric method agree satisfactorily with those obtained in the hospital. The biggest relative standard deviation (RSD) is $2.4 \%$ for the three independent measurements, demonstrating good reproducibility of the method. The above results imply that the proposed system can be applicable for reliable determinations of glucose in human blood samples.

\section{Conclusions}

In summary, we have testified that umbelliferone, a kind of coumarin derivative, could act as a novel type of peroxidase mimic to catalyze the oxidation of the peroxidase substrate TMB in the presence of $\mathrm{H}_{2} \mathrm{O}_{2}$ to generate a blue product. The catalytic activity of umbelliferone was dependent on the $\mathrm{pH}$, reaction temperature, concentration of $\mathrm{H}_{2} \mathrm{O}_{2}$ and incubation time. On this basis, a convenient, brief and sensitive $\mathrm{H}_{2} \mathrm{O}_{2}$ and glucose detection method was successfully established. $\mathrm{H}_{2} \mathrm{O}_{2}$ can be detected with a linear range from 1.0 to $100.0 \mu \mathrm{M}$ and a detection limit of $0.5 \mu \mathrm{M}$. Coupled with glucose oxidase, a linear range from 5.0 to $100.0 \mu \mathrm{M}$ and a detection limit of $2.5 \mu \mathrm{M}$ was obtained for glucose detection. What is more, the demonstrated colorimetric method was applied to glucose determination in human serum, and the values of the glucose levels agree very well with that obtained in hospitals, exhibiting good accuracy and reproducibility. This work not only provides some new insights into seeking for novel molecular enzyme mimetics with excellent catalytic activities, but also expands the application of molecular enzyme mimetics as a substitute of peroxidase-like catalysts.

\section{Acknowledgements}

This work was financially supported by the National Natural Science Foundation of China (Nos. 21405124, 21175110), the Fundamental Research Funds for the Central Universities (No. XDJK2013A022). 


\section{Supporting Information}

This material is available free of charge on the Web at http:// www.jsac.or.jp/analsci/.

\section{References}

1. I. X. Green, W. Tang, M. McEntee, M. Neurock, and J. T. Yates, Jr., J. Am. Chem. Soc., 2012, 134, 12717.

2. H. G. Yang, J. Q. Zha, P. Zhang, Y. H. Xiong, L. J. Su, and F. G. Ye, $R S C A d v$., 2016, 6, 66963.

3. J. Z. Chen, Y. J. Liu, G. X. Zhu, and A. H. Yuan, Cryst. Res. Tenchol., 2014, 49, 309.

4. R. Akter, C. K. Rhee, and M. A. Rahman, Biosens. Bioelectron., 2015, 66, 539.

5. L. J. Su, Y. H. Xiong, H. H. Yang, P. Zhang, and F. G. Ye, J. Mater. Chem. B, 2016, 4, 128.

6. L. Z. Gao, J. Zhuang, L. Nie, J. B. Zhang, Y. Zhang, N. Gu, T. H. Wang, J. Feng, D. L. Yang, S. Perrett, and X. Y. Yan, Nat. Nanotechnol., 2007, 2, 577.

7. E. Nakamura and H. Isobe, Acc. Chem. Rev., 2003, 36, 807.

8. Y. N. Ding, B. C. Yang, H. Liu, Z. X. Liu, X. Zhang, X. W. Zheng, and Q. Y. Liu, Sens. Actuators, B, 2018, 259, 775.

9. Y. F. Wang, N. Pan, and C. F. Peng, Anal. Sci., 2017, 33, 321.

10. Q. Y. Liu, Y. T. Yang, H. Li, R. R. Zhu, Q. Shao, S. G. Yang, and J. J. Xu, Biosens. Bioelectron., 2015, 64, 147.

11. Q. Y. Liu, Y. T. Yang, X. T. Lv, Y. N. Ding, Y. Z. Zhang, J. J. Jing, and Ch. X. Xu, Sens. Actuators, B, 2017, 240, 726.

12. Z. Z. Yang, F. Q. Ma, Y. Zhu, S. H. Chen, C. Wang, and X. F. Lu, Dalton Trans., 2017, 46, 11171.

13. F. Kang, X. S. Hou, and K. Xu, Nanotechnology, 2015, 26, 405707

14. E. Kuah, S. Toh, J. Yee, Q. Ma, and Z. Q. Gao, Chemistry, 2016, 22, 8404.

15. L. Liu, Y. Shi, Y. F. Yang, M. L. Li, Y. J. Long, Y. M. Huang, and H. Z. Zheng, Chem. Commun., 2016, 52, 13912.

16. R. Simkovitch and D. Huppert, J. Phys. Chem. B, 2015 119,14683

17. F. Wu and S. Sheu, Chin. Pharm. J., 1992, 44, 257.
18. C. M. Krauter, J. Mohring, T. Buckup, M. Pernpointner, and M. Motzkus, Phys. Chem. Chem. Phys., 2013, 15, 17846.

19. C. C. Perry, V. J. Tang, K. M. Konigsfeld, J. A. Aguilera, and J. R. Milligan, J. Phys. Chem. B, 2011, 115, 9889.

20. W. Y. Zhai, C. X. Wang, P. Yu, Y. X. Wang, and L. Q. Mao, Anal. Chem., 2014, 86, 12206.

21. L. Pan, X. Z. Li, Z. Q. Yan, H. R. Guo, and B. Qin, Plant Physiol. Biochem., 2015, 97, 272.

22. B. Garg and T. Bisht, Molecules, 2016, 21.

23. S. Kandil, A. D. Westwell, and C. McGuigan, Bioorg. Med. Chem. Lett., 2016, 26, 2000.

24. P. D. Josephy, T. Eling, and R. P. Mason, J. Biol. Chem., 1982, 257, 3669.

25. Z. Z. Yang, Y. Zhu, G. D. Nie, M. X. Li, C. Wang, and X. F. Lu, Dalton Trans., 2017, 46, 8942.

26. H. H. Zhi, J. D. Wang, S. J. Wang, and Y. J. Wei, J. Spectrosc., 2013, Article ID 147128.

27. J. Zhang, C. G. Liu, and Y. J. Wei, Ниахие Tongbao, 2011 $74,957$.

28. P. Ju, Y. Z. Yu, M. Wang, Y. Zhao, D. Zhang, C. J. Sun, and X. X. Han, J. Mater. Chem. B, 2016, 4, 6316.

29. Y. F. Yang, D. J. Shen, Y. J. Long, Z. X. Xie, and H. Z. Zheng, Sci. Rep., 2017, 7, 43141.

30. L. Su, W. Qin, H. Zhang, Z. U. Rahman, C. Ren, S. Ma, and X. Chen, Biosens. Bioelectron., 2015, 63, 384.

31. H. Wei and E. Wang, Anal. Chem., 2008, 80, 2250.

32. F. M. Qiao, L. J. Chen, X. N. Li, L. F. Li, and S. Y. Ai, Sens. Actuators, B, 2014, 193, 255.

33. Q. Chen, J. Chen, C. J. Gao, M. L. Zhang, J. Y. Chen, and H. D. Qiu, Analyst, 2015, 140, 2857.

34. C. M. Riccardi, D. Mistri, O. Hart, M. Anuganti, Y. Lin, R. M. Kasi, and C. V. Kumar, Chem. Commun., 2016, 52, 2593.

35. Z. Q. Yan, D. D. Wang, H. Y. Cui, D. H. Zhang, Y. H. Sun, H. Jin, X. Z. Li, X. Y. Yang, H. R. Guo, X. F. He, L. Pan, X. Ren, K. Guo, and B. Qi, Acta Physiol. Plant, 2016, 38 , 248.

36. L. Su, X. A. Yu, W. J. Qin, W. P. Dong, C. K. Wu, Y. Zhang, G. J. Mao, and S. L. Feng, J. Mater. Chem. B, 2017, 5, 116.

37. S. H. Lim, J. Wei, J. Lin, Q. Li, and K. Jin, Biosens. Bioelectron., 2005, 11, 2341 . 